\title{
Mercados de la UNAD en el Ecuador, de acuerdo con las apuestas productivas
}

\section{Resumen}

El artículo analiza la importancia que tiene la identificación de nichos de mercado para los programas académicos de la UNAD en Ecuador, mediante el análisis de las apuestas productivas y de la evolución de los acuerdos comerciales o tratados firmados entre Colombia y Ecuador en materia educativa, como los estudios realizados en Ecuador sobre población potencial de estudiantes de este país en relación con la educación superior. La investigación obtuvo resultados interesantes sobre diferentes universidades e instituciones de educación superior de Ecuador con las cuales existen condiciones para definir acuerdos, alianzas o consorcios con la UNAD, de Colombia.

Palabras clave: Nichos de mercados, apuestas productivas, internacionalización educativa.

\section{Abstract}

The article discusses the importance of identifying niche markets for the academic programs of the UNAD in Ecuador, through the analysis of productive bets, the evolution of trade agreements or treaties between Colombia and Ecuador in education, as studies in Ecuador on potential student population of this country in relation to higher education. The investigation had interesting results on different universities and institutions of higher education in Ecuador with which there are conditions for the establishment of agreements, alliances or consortia with the UNAD, Colombia.

Key words: Niche markets, betting productive, education internationalization.

Recibido: 12 de abril de 2012

Aprobado: 28 de julio de 2012

\footnotetext{
1 Administrador de empresas, Universidad Nacional Abierta y a Distancia (UNAD). Especialista en gerencia estratégica de mercadeo, Universidad Nacional Abierta y a Distancia (UNAD). Máster en administración de empresas, UNAD Florida. Universidad Nacional Abierta a Distancia (UNAD) CEAD Santa Marta. Coordinador de investigación. Coordinador de desarrollo regional y proyección comunitaria, Santa Marta (Colombia). jesus.fandino@unad.edu.coa
} 


\section{Introducción}

Este artículo es el resultado del proyecto de investigación Identificación de Nichos de Mercados en Ecuador para los programas académicos de la UNAD, presentado a la UNAD Florida.

Los nichos de mercados son grupos más pequeños que los segmentos de mercados (Kotler, 2001) y se relacionan con mercados más reducidos, cuyas necesidades no están bien atendidas, lo cual convierte este concepto en una herramienta fundamental del marketing para detectar necesidades o deseos específicos o parecidos con el mercado educativo de Colombia, y, en especial, a los programas académicos de la UNAD.

La globalización en el nuevo milenio ha permitido que se creen economías de mercado promotoras de la mercantilización de los servicios de educación, no solo como estrategia de comercialización, sino como extensión de procesos de ciencia, tecnología e innovación entre países. Lo anterior, como consecuencia del aumento de nuevos proveedores, la exportación de servicios educativos y el aumento de la imposición de expansión del mercado que ha impactado la educación superior.

La exportación de servicios es un referente complementario para consolidar la educación superior en el escenario internacional; por tal razón, las universidades colombianas deben promover la exportación de servicios educativos, con el apoyo del Gobierno, para ampliar la oferta a otros países. Colombia ha llegado a acuerdos comerciales con varios países de características similares a las propias, a través de tratados de zonas de libre comercio que permiten extender la oferta de servicios educativos de carácter superior a otras regiones.

La UNAD, como ente estatal, no escapa de esta realidad global, pues necesita fortalecer su oferta educativa en el ámbito internacional, mediante el desarrollo de investigaciones de mercado que permitan identificar posibles mercados, en países con características socioeconómicas y culturales similares a las de Colombia. De esta forma, la UNAD busca expandir sus servicios y dar respuesta a su proceso de internacionalización.

Por todo lo expuesto, el fin del artículo es identificar posibles mercados para la UNAD en el Ecuador, según las apuestas productivas, con el propósito de proyectar la internacionalización de la institución, a partir de una revisión documental sobre los principales elementos teóricos relacionados con nuevos mercados.

Para lograr tal objetivo, se realiza una revisión documental, con el fin de describir las apuestas productivas de Ecuador que permitan identificar posibles mercados para los programas educativos de la UNAD, identificar las universidades o instituciones de educación supe- rior con metodologías similares a las de la UNAD el en Ecuador que permitan definir convenios, alianzas o consorcios viables y analizar los acuerdos de comercialización de servicios educativos contenidos en tratados relativos a Ecuador y Colombia. Los resultados están vinculados a cada uno de estos aspectos, y en las conclusiones se presentan los posibles mercados como estrategia fundamental para identificar los principales factores que tener en cuenta para la oferta de programas académicos en un país del exterior.

\section{Métodos}

El tipo de estudio es exploratorio. El método de estudio es una revisión documental en fuentes secundarias, con un análisis cuantitativo. Las técnicas y los procedimientos para la recolección de datos con el fin de desarrollar esta investigación es la observación documental, la cual se realizó a través de una revisión donde se analizaron textos escritos por autores que han trabajado anteriormente con el tema, y utilizando una ficha de contenido para tratar de identificar, describir y analizar los posibles programas académicos que, a juzgar por las características educativas de la UNAD, puedan ser pertinentes y admitidos en Ecuador. Por tanto, se requiere el desarrollo de una investigación de mercado que permita identificar los programas pertinentes de la oferta educativa de la UNAD que no se oferten en Ecuador, en la modalidad a distancia y virtual.

Las variables de estudio en la presente investigación son:

- Número de apuestas productivas del Ecuador que permitan sugerir la oferta educativa de la UNAD.

- Cantidad de universidades o instituciones de educación superior con metodología similares a la de la UNAD en Ecuador que permitan definir convenios, alianzas o consorcios viables.

- Número de acuerdos comerciales de servicios educativos firmados en tratados entre Ecuador y Colombia.

- De acuerdo con la investigación documental que se va a realizar, se analizan las fuentes secundarias necesarias para la llevar a cabo el trabajo; entre ellas se pueden citar diversos tipos de documentos:

- Documentos secundarios: boletines de resúmenes (analíticos, indicativos, índices de citas), bibliografías, repertorios y directorios. Se los ha utilizado con base en información seleccionada de sitios web, como se describirá en el siguiente punto.

- Documentación informatizada: Internet, catálogos en línea, buscadores, bases de datos especializadas, etc. Dentro de las que se destacan las siguientes páginas del Ecuador: la página del Instituto Nacional de Esta- 
dísticas y Censos del Ecuador; el sitio web del Instituto Internacional para la Educación Superior en América Latina y el Caribe (IESALC), que es un organismo de la Organización de las Naciones Unidas para la Educación, la Ciencia y la Cultura (UNESCO) dedicado a promover la educación superior; la página del Consejo Nacional de Educación del Ecuador, y la página del Ministerio de Educación del Ecuador.

- Se ha analizado un total de 5 investigaciones relacionadas con la educación superior en Ecuador, así como
16 proyectos de investigación sobre nuevos proveedores de educación superior en otros países de América; algunos de estos últimos relacionaron las URL de Internet con la respectiva fecha cuando se las consultó.

\section{Resultados}

Los sectores productivos de Ecuador se resumen en la Tabla 1, de acuerdo con la estructura de la población según el sector productivo de empleo.

Tabla 1. Estructura de la población según sector productivo de empleo (2001)

\begin{tabular}{|l|r|}
\hline \multicolumn{1}{|c|}{ Sector productivo } & \multicolumn{1}{|c|}{ Proporción (\%) } \\
\hline Agricultura & 8 \\
\hline Industria & 24 \\
\hline Servicios & 68 \\
\hline
\end{tabular}

Fuente: Ministerio de Fomento, Industria y Comercio (2010).

De los sectores mencionados se clasifican las apuestas productivas, de las cuales, a suvez, se destacan 8 subsectores que generan el $72 \%$ del empleo en Ecuador; la inci- dencia en el PIB es muy alta, $y$, al mismo tiempo, refuerza la oferta exportadora de este país. Dichos subsectores se relacionan en la Tabla 2.

Tabla 2. Sectores económicos de Ecuador (1998)

\begin{tabular}{|l|}
\hline \\
\hline Agricultura, caza, silvicultura y pesca \\
\hline Petróleo y minas \\
\hline Industria manufacturera \\
\hline Electricidad, gas y agua \\
\hline Construcción \\
\hline Comercio y hoteles \\
\hline Transporte y comunicación \\
\hline Servicios financieros \\
\hline
\end{tabular}

Fuente: SICA-MAG (2009).

De acuerdo con el sector servicios, con el $68 \%$ en la Tabla 1, y estos subsectores que proveen el mayor porcentaje de empleo, se establece una relación directa, donde se observa la oferta de los programas académicos quetienenlamayorpoblaciónestudiantilenestepaís(como
Administración y Comercio [30,38\%], Ciencias de la Salud [20,57\%], Ciencias Sociales [15,71\%] y los programas de pregrado en Educación [14,84\%]. Los programas de tecnología solo representan un $8,77 \%$, y los de Agrícola y Pecuaria, un $4,86 \%)$. 
Tabla 3. Informe de educación superior por áreas en Ecuador (2006)

\begin{tabular}{|l|r|}
\hline \multicolumn{1}{|c|}{ Áreas } & \multicolumn{1}{|c|}{ Proporción (\%) } \\
\hline Administración y Comercio & 30,38 \\
\hline Agrícola y Pecuaria & 4,86 \\
\hline Artes y Arquitectura & 0,87 \\
\hline Ciencias Básicas & 3,47 \\
\hline Ciencias de la Salud & 20,57 \\
\hline Ciencias Sociales & 15,71 \\
\hline Educación & 14,84 \\
\hline Humanidades y Ciencia de la Cultura & 0,52 \\
\hline Tecnología & 8,77 \\
\hline
\end{tabular}

Fuente: Archivos del Consejo Nacional de Educación Superior (CONESUP).

El informe de los institutos superiores técnicos y tecnológicos por áreas de formación en Ecuador durante 2006 muestra que las carreras de administración y comercio son las de mayor demanda, con un 40,72\%, al igual que en las instituciones de educación superior; sin embargo, las carreras de tecnología se ubican en segundo lugar, con un 30,25\%, a diferencia de los resultados en educación superior, como se observa en la Tabla 3.

Tabla 4. Informe de institutos superiores técnicos y tecnológicos por áreas en Ecuador (2006)

\begin{tabular}{|l|r|}
\hline \multicolumn{1}{|c|}{ Áreas } & \multicolumn{1}{|c|}{ Proporción (\%) } \\
\hline Administración y Comercio & 40,72 \\
\hline Agrícola y Pecuaria & 5,87 \\
\hline Artes y Arquitectura & 9,86 \\
\hline Ciencias Básicas & 0,47 \\
\hline Ciencias de la Salud & 1,35 \\
\hline Ciencias Sociales & 4,46 \\
\hline Educación & 6,28 \\
\hline Humanidades y Ciencia de la Cultura & 0,74 \\
\hline Tecnología & 30,25 \\
\hline
\end{tabular}

Fuente: Archivos del Consejo Nacional de Educación Superior (CONESUP).

Comparando estos resultados con la preferencia que tienen los trabajadores de los sectores servicios e industrias y los habitantes de Ecuador por determinados programas educativos que satisfacen sus expectativas en sectores de mayor demanda de empleo, como el sector de servicios, que absorbe el $68 \%$ del empleo de Ecuador, y la industria, con un $24 \%$, lo que constituye información fundamental para la selección de programas como administración y comercio, ciencias de la salud, ciencias sociales y de la educación. De esta forma los trabajadores de los sectores de servicios, comercio, social y de educación, representan para Kotler (1996) un nicho de mercado ideal, que tiene el tamaño suficiente como para ser rentable.

La relación de la economía con los programas académicos que ofertan las instituciones de educación superior e institutos superiores técnicos y tecnológicos en el Ecuador es similar en cuanto a la proporción de personal empleado por actividad en este país para el periodo 2000-2005. En efecto, hay durante ese lapso una alta proporción de pertinencia de los programas académicos con el personal empleado por actividad económica. 
Tabla 5. Proporción de población económicamente activa de 2000 a 2005, según rama de actividad

\begin{tabular}{|c|c|c|c|c|c|c|}
\hline Rama de actividad & $\begin{array}{c}2000 \\
(\%)\end{array}$ & $\begin{array}{c}2001 \\
(\%)\end{array}$ & $\begin{array}{c}2002 \\
(\%)\end{array}$ & $\begin{array}{c}2003 \\
(\%)\end{array}$ & $\begin{array}{c}2004 \\
(\%)\end{array}$ & $\begin{array}{c}2005 \\
(\%)\end{array}$ \\
\hline $\begin{array}{l}\text { Agricultura, ganadería } \\
\text { y caza }\end{array}$ & 7,0 & 6,5 & 7,5 & 7,8 & 8,3 & 6,6 \\
\hline Pesca y criaderos & 1,5 & 1,2 & 1,0 & 1,3 & 1,0 & 1,0 \\
\hline $\begin{array}{l}\text { Explotación de minas y } \\
\text { canteras }\end{array}$ & 0,6 & 0,5 & 0,6 & 0,6 & 0,4 & 0,4 \\
\hline $\begin{array}{l}\text { Industria } \\
\text { manufacturera }\end{array}$ & 15,6 & 16,6 & 14,5 & 13,8 & 14,0 & 14,2 \\
\hline $\begin{array}{l}\text { Suministro de electric- } \\
\text { idad, gas y agua }\end{array}$ & 0,6 & 0,8 & 0,4 & 0,5 & 0,6 & 0,6 \\
\hline Construcción & 7,1 & 6,4 & 7,0 & 6,8 & 6,4 & 5,7 \\
\hline $\begin{array}{l}\text { Comercio, reparación } \\
\text { de vehículos y efectos } \\
\text { personales }\end{array}$ & 26,6 & 28,0 & 28,1 & 28,3 & 28,4 & 29,0 \\
\hline Hoteles y restaurantes & 4,3 & 4,3 & 4,2 & 3,7 & 4,4 & 5,4 \\
\hline $\begin{array}{l}\text { Transporte, almace- } \\
\text { namiento } \\
\text { y comunicación }\end{array}$ & 6,3 & 6,7 & 6,4 & 6,6 & 6,9 & 7,0 \\
\hline $\begin{array}{l}\text { Intermediación finan- } \\
\text { ciera }\end{array}$ & 1,3 & 0,9 & 1,3 & 1,5 & 1,3 & 1,2 \\
\hline $\begin{array}{l}\text { Act. inmobiliarias, em- } \\
\text { presariales } \\
\text { y alquiler }\end{array}$ & 4,0 & 4,3 & 4,5 & 4,4 & 4,9 & 4,8 \\
\hline $\begin{array}{l}\text { Administración pública } \\
\text { y defensa; seguridad } \\
\text { social }\end{array}$ & 4,1 & 4,4 & 4,2 & 5,1 & 4,5 & 4,7 \\
\hline Enseñanza & 6,6 & 5,8 & 6,9 & 6,6 & 6,8 & 7,4 \\
\hline $\begin{array}{l}\text { Act. servicios sociales } \\
\text { y de salud }\end{array}$ & 3,2 & 2,7 & 3,4 & 3,3 & 3,6 & 3,2 \\
\hline $\begin{array}{l}\text { Otras actividades co- } \\
\text { munitarias } \\
\text { sociales y personales }\end{array}$ & 4,9 & 4,2 & 3,5 & 4,9 & 4,6 & 4,5 \\
\hline $\begin{array}{l}\text { Hogares privados con } \\
\text { servicio } \\
\text { doméstico }\end{array}$ & 6,4 & 6,3 & 6,2 & 4,6 & 3,9 & 4,4 \\
\hline $\begin{array}{l}\text { Organizaciones extra- } \\
\text { territoriales }\end{array}$ & 0,0 & 0,0 & 0,0 & 0,0 & 0,0 & 0,0 \\
\hline No específica & & 0,5 & 0,0 & 0,0 & 0,0 & 0,0 \\
\hline
\end{tabular}

Fuente: Instituto Nacional de Estadísticas y Censos (INEC) (s. f.).

Cabe observar que el sector con mayor demanda es el de comercio, reparación de vehículos y efectos personales, con un $29 \%$, en promedio, de la población económicamente activa; ello demuestra una fuerte relación con los análisis anteriores.

Los servicios, con un $68 \%$, tienen una buena predisposición por parte de los empleados de este sector a la hora de elegir programas afines a este renglón de la economía ecuatoriana, y donde se destacan administración y comercio, educación, ciencias de la salud y sociales. No obstante, también sobresalen otras actividades que presentan dinámicas de crecimiento importantes, como la industria manufacturera, con un $15 \%$, en promedio, y la agricultura, ganadería y caza, con un promedio del $7,5 \%$, y en las cuales las universidades 0 los institutos superiores técnicos y tecnológicos pueden estar enfocados en nichos de mercados muy especí- 
ficos de trabajadores de la industria manufacturera y agricultura, por los programas de tecnología.

Otro interesante estudio es el comparativo entre las ramas de actividad por grupos ocupacionales y el nivel de formación, donde se destaca el personal directivo perteneciente al sector público y privado, que presenta un incremento, a 2005 , del $170 \%$; los técnicos y profesionales de nivel medio presentan un incremento del $140 \%$; los trabajadores de los servicios y comerciantes, del $125 \%$; los profesionales científicos e intelectuales, del $120 \%$, y los empleados de oficina, del $117 \%$.
De este análisis se infiere que los programas de administración y comercio, educación, ciencias de sociales y de la salud, representativos para los trabajadores de nivel directivo del sector público y privado, técnicos y profesionales de nivel medio, trabajadores de los servicios y comerciantes como los empleados de oficinas.

Lo que hace de estos programas nichos de mercados con mayor demanda para satisfacer necesidades educativas entre los trabajadores citados en el párrafo anterior.

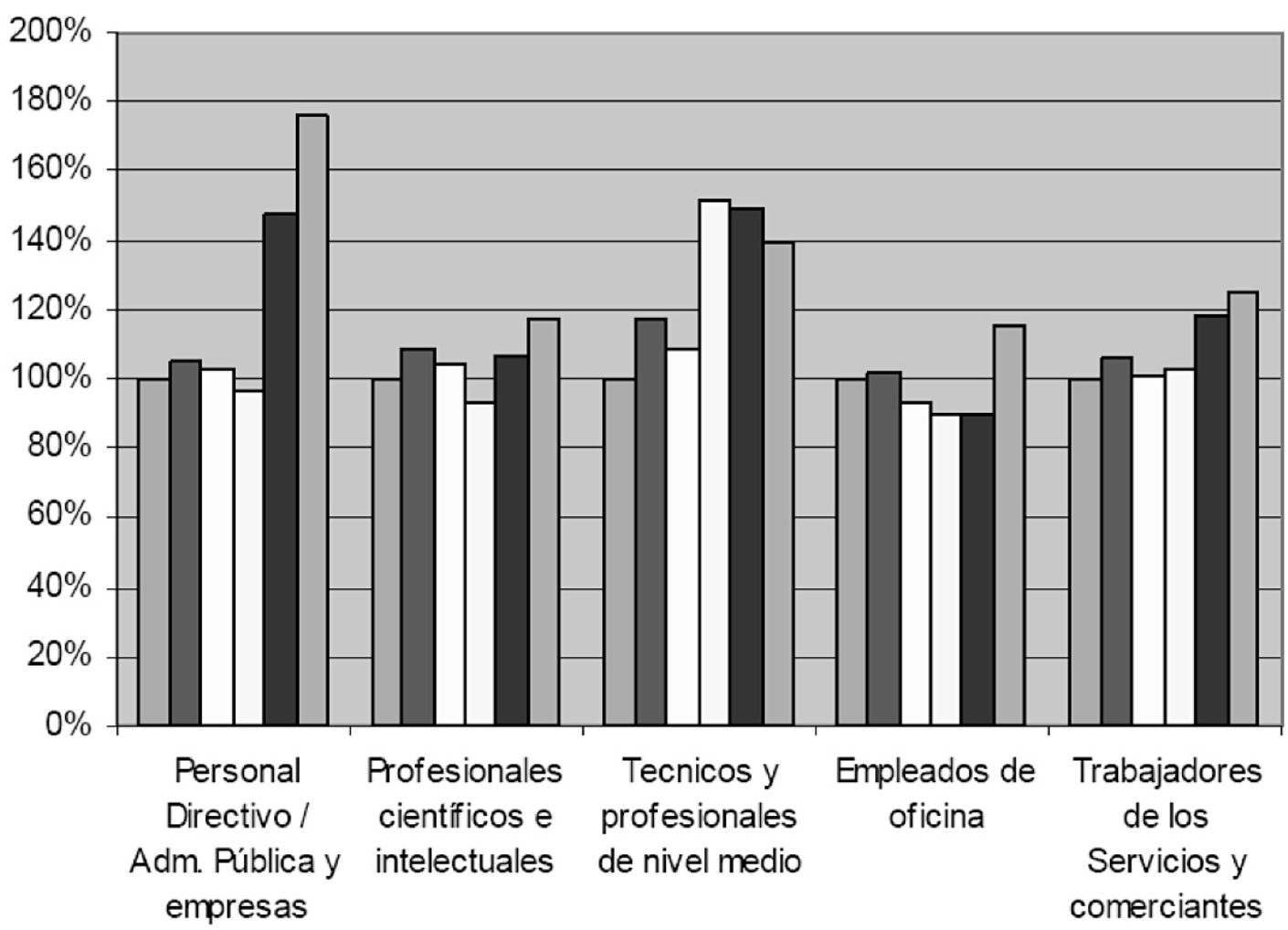

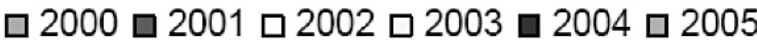

Grafica 1. Índice de ocupación personal empleado por actividad de 2000 a 2005 (área urbana de Ecuador) Fuente: análisis y proyección de la población económicamente activa (PEA) del Ecuador-INEC.

Otros segmentos de mercado son los trabajadores calificados agropecuarios y pesqueros, quienes, aunque en estudio, tienen un descenso entre 2004 y 2005 del $160 \%$ al $120 \%$; son un grupo o nicho de mercado para los programas de tecnología. Así mismo, los operarios de instalaciones, maquinarias y montadoras; aunque se observa un segmento de operarios no calificados, pueden ser consumidores con necesidades y características homogéneas, iguales a las de los trabajadores calificados. Desde este punto de vista, los programas de tecnología, por su variedad temática, se convierten en una gran estrategia de crecimiento para las universidades y los institutos superiores técnicos y tecnológicos, como se observa en la Gráfica 2. 


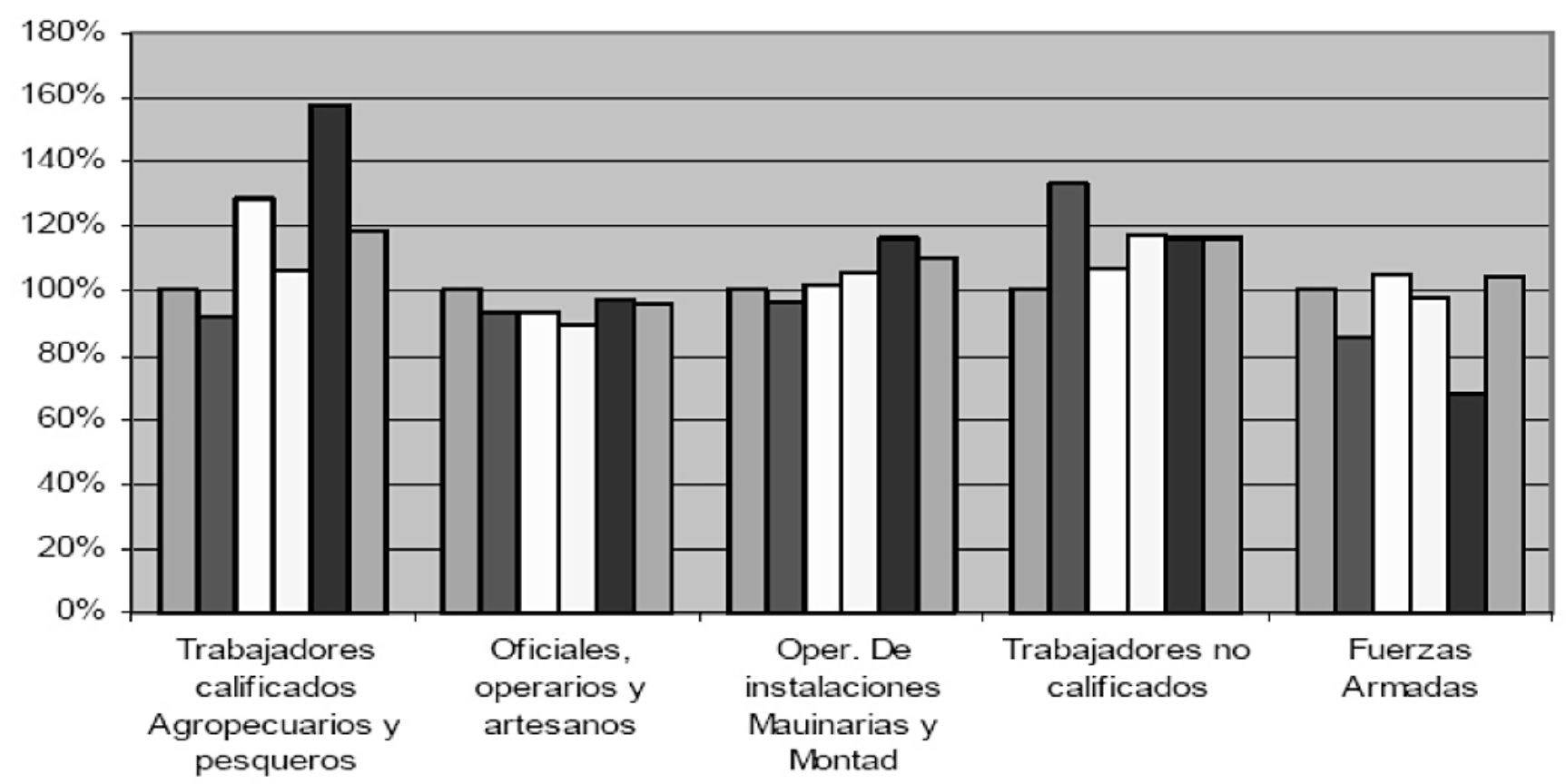

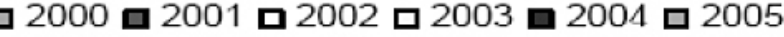

\section{Grafica 2. Índice de ocupación de personas empleadas por actividad en el área urbana de Ecuador (2000-2005), según grupo de ocupación}

Fuente: análisis y proyección de la población económicamente activa (PEA) del Ecuador-INEC.

En Ecuador hay un total de 67 universidades y 8 escuelas politécnicas reconocidas por el Estado ecuatoriano. Igualmente, 380 institutos superiores técnicos y tecnológicos, que también se amparan en la Ley de Educación Superior e integran el Sistema de Educación Superior. De estas universidades, el 27\% prestan servicios a través de la modalidad a distanciaonline, y el $73 \%$, de forma presencial; algunas de ellas pueden incluir la modalidad semipresencial. De esta forma se tiene que existen 19 universidades; 3 de ellas, con registro nacional de acreditación, y con las cuales se puede realizar al menos un convenio, alianza o consorcio, pues pueden facilitar la oferta de los programas de la UNAD en Ecuador, de acuerdo con datos del CONESUP (2009).

De esta forma, es necesario revisar la oferta de programas a distancia que son de mayor demanda para los nichos de mercado identificados en el estudio, pues lo que se busca es identificar posibles mercados para los programas educativos de la UNAD en Ecuador; más aun, porque el porcentaje de universidades que cuentan con programas a distancia es muy representativo en este país, lo que marca una tendencia para crear competencia entre las universidades locales con las universidades extranjeras, como la UNAD.

Este porcentaje se refleja en estudios realizados durante 2002 , según los cuales en Ecuador el $72 \%$ de las universidades contaban con programas presenciales y a distancia; el 24\%, con programas presenciales, y el $4 \%$, con semipresenciales, lo que representa un dato muy importante, pues dichas universidades, casualmente, irán cada día apoyando más con tecnología sus programas académicos a distancia tradicionales (UNESCO, 2004).

De esta forma se realiza un análisis de la oferta virtual y a distancia de pregrado con las universidades de Ecuador más representativas de esta modalidad, iniciando con la Escuela Politécnica Nacional, la cual en ciencias de la educación ofrece Física y Matemáticas; en Ciencias Administrativas, el Programa de Ingeniería Empresarial; en Ciencias Básicas e Ingenierías, los programas de Ingeniería Ambiental, Ingeniería Electrónica, Ingeniería Electrónica y de Control de Redes, Ingeniería Electrónica y Telecomunicaciones, Ingeniería en Sistemas Informáticos y de Computación, Ingeniería Química, e Ingeniería Agroindustrial. En tecnologías ofrece Tecnología en Electrónica y Telecomunicaciones, y Tecnología en Mantenimiento Industrial, entre otras. Con esta institución la UNAD podría considerar un convenio para la Licenciatura en Inglés como Lengua Extranjera; en el plano de ingeniería, la de Alimentos e Ingeniería Industrial, con los cuales se respondería a los sectores económicos de mayor desarrollo en el Ecuador, como lo son servicios e industrias. 
La segunda institución en estudio es la Universidad Católica de Santiago de Guayaquil, la cual lleva 50 años de existencia y ofertando programas a distancia, entre los cuales se destacan Administración de Empresas, Licenciatura en Educación Básica, Trabajo Social e Ingeniería en Contabilidad y Auditoría, como programas afines con la UNAD; sin embargo, se podrían ofertar a través de ella programas como Psicología, Comunicación Social, ingenierías como las de Sistemas, Industrial, de Telecomunicaciones, y Electrónica, y las tecnologías de estos programas profesionales, así como la Tecnología en Gestión Comercial y de Negocios, y las licenciaturas de Filosofía y de Etnoeducación, programas con todos los cuales se respondería a los nichos de mercados de trabajadores pertenecientes a los sectores económicos o a apuestas productivas más dinámicas de Ecuador.

La tercera institución es la Facultad Latinoamericana de Ciencias Sociales (FLACSO) Ecuador, la cual oferta los programas académicos de Antropología, Economía y Sociología, más relacionados con la UNAD. Con esta institución se podría llegar a un convenio o alianza para la oferta de programas como Psicología, Filosofía y Comunicación Social, lo que respondería a trabajadores del sector servicio en lo social y en salud.

La Escuela Politécnica del Ejército (ESPE) tiene licenciaturas en Educación Infantil, además de Tecnología en Computación, Tecnología en Administración Microempresarial, e Ingeniería Comercial, programas académicos que no tienen relación con los de la UNAD, aunque esta sí podría llegar a un acuerdo para fortalecer su oferta académica.

La Universidad de Cuenca, en las modalidades a distancia y virtual, ofrece Administración de Empresas, Ingeniería Electrónica y de Comunicaciones, Psicología, Medicina Veterinaria y Zootecnia, e Ingeniería Agronómica. Es la universidad más afín a la UNAD, y con la cual se podrían realizar intercambios de movilidad, para compartir experiencias que puedan llegar a la oferta de posgrados en alianza.

Para llegar a estas alianzas con universidades ecuatorianas la UNAD puede acudir al Tratado de Asociación Latinoamericana de Integración (ALADI), firmado en Montevideo (1980), y cuyo propósito es el establecimiento, de forma gradual y progresiva, de un mercado común latinoamericano para acciones de cooperación en materia científica y tecnológica, así como de cooperación e intercambio de bienes en las áreas cultural, educacional y científica, y de superación de obstáculos técnicos para el comercio. Desde este tratado se podrían celebrar convenios de cooperación para el intercambio de bienes en las áreas culturales, educacionales y científicas, a través del extenso marco de cooperación educativa, cultural y científica de los países asociados, como Colombia y Ecuador, para mejorar y elevar los niveles de instrucción, capacitación y conocimiento recíproco de los pueblos de la región.

Otro acuerdo con ayuda del cual la UNAD podría celebrar alianzas con universidades ecuatorianas es el del Grupo de Río, creado para la cooperación educativa, y el cual incluye dentro de sus campos de acción aligerar la formación progresiva de un mercado tradicional de bienes culturales y educativos que facilite la libre circulación en los territorios de los países miembros, y, transitoriamente, a los demás países latinoamericanos y del Caribe.

A ese respecto se destacan los siguientes acuerdos en materia educativa: la responsabilidad de Ecuador con la integración regional, mediante la incorporación de este país al Protocolo de Integración Educativa y Reconocimiento de Certificados, Títulos y Estudios de Nivel Primario y Medio no Técnico, entre los países integrantes del Mercosur, del cual hace parte Colombia, y donde, además, se firma un memorando de alcance sobre la instauración y la puesta en funcionamiento de un sistema de acreditación de carreras universitarias para el reconocimiento regional de la calidad académica, relacionado con las titulaciones que se den a través del Mercosur y sus países asociados. Dicho documento permite la movilidad de personas entre los países de la región y avala los mecanismos regionales de reconocimiento de títulos y diplomas universitarios (Mercosur Educativo, 2009).

Por otra parte, la UNESCO firmó un acuerdo similar sobre reconocimientos de título y diplomas, a través del Comité Regional de América Latina y el Caribe (CRALC), mediante el Convenio Regional de Convalidación de Estudios, Títulos y Diplomas de Educación Superior para América Latina y el Caribe, firmado por 18 países, y de los cuales 13 pertenecen a la región y otros 5 , a otras zonas geográficas; entre estos últimos se destacan Colombia y Ecuador. Con estos 3 acuerdos la UNAD puede explorar convenios de cooperación o alianzas con universidades de Ecuador, y así podría llegar a los nichos de mercados citados en el estudio.

Estas alianzas o convenios se pueden dar debido a que en Ecuador las universidades trabajan sus procesos académicos mediante la unidad de crédito académico, la cual se distribuye entre la población por nivel Técnico Superior (con el $8 \%$ ), Pregrado (de tercer nivel, con el $89,5 \%)$ y Postgrado (2,5\%). El número de créditos para optar al título se dividen en: Técnico, con 120; Tecnólogo, con 185, y Pregrado completo, con 240. Los créditos académicos para diploma superior son 15; para Especialista, 30; para Magíster, 60, y para Doctorado, 90, según el CONESUP (2006). 
La cantidad de créditos establecidos en Ecuador no son equivalentes a los establecidos por la UNAD de Colombia: por ejemplo, los créditos exigidos en Ecuador para la carrera de Psicología son 160 créditos; para Comunicación, 160, y para Filosofía, 160; es decir, en todos los casos enunciados están por debajo de los 240; sin embargo cabe anotar que dicha diferencia se da porque en Ecuador las horas de un crédito académico oscilan de 25-30, lo cual, desde el punto de vista de una alianza, favorece a las universidades ecuatorianas para acogerse, a través de un tratado, a los programas educativos que serían más atractivos en Ecuador, por su corta duración. No obstante, dichas ventajas o desventajas deben ser estudiadas a la luz de leyes o acuerdos de los 2 países (CONESUP, 2006).

La suscripción de acuerdos para la libre oferta de servicios educativos requiere que los países asociados cuenten con una infraestructura tecnológica y de conectividad adecuadas para la exportación de programas académicos a los países agrupados en el acuerdo. Frente a tal requerimiento Ecuador presenta una infraestructura tecnológica y de conectividad con el índice más bajo de avance de Internet en América Latina; no obstante, a lo largo de los últimos 3 años tanto la oferta como la demanda se han elevado de forma sostenida, provocado ello por el fenómeno global de la incorporación y la utilización de Internet en la empresas y las universidades ecuatorianas; principalmente, en las empresas proveedoras de acceso a Internet y de telefonía fija, que durante los últimos años han digitalizado gran parte de su infraestructura (UNESCO, 2004).

En Ecuador las universidades con acceso a Internet cuentan con servidores de Internet y correo electrónico, respaldadas por sus correspondientes páginas web. La cantidad de servidores es de 1-5, teniendo en cuenta el número de estudiantes que tengan. En referencia a la infraestructura interna, el $52 \%$ de las universidades cuenta con fibra óptica; especialmente, las que disponen de un mayor número de estudiantes, y cuya carga de trabajo en sus redes internas, por lo tanto, es mayor (UNESCO, 2004).

También durante 2001 y 2002, otras universidades ofertaron carreras de pregrado a distancia con apoyo de Internet, como la Escuela Superior Politécnica del Ejército (ESPE), la Universidad de las Américas (UDLA) y la Universidad San Francisco de Quito (USFQ), la cual se destaca porque cuenta con 8 carreras mediadas por tecnologías; cada carrera se forma de cursos, y cada curso requiere una carga de trabajo de 45 horas, de las cuales 9 son presenciales, al igual que las evaluaciones (UNESCO, 2004).

Para el caso de programas académicos totalmente en línea, en 1999 la Universidad Técnica Particular de Loja
(UTPL) lanzó el curso Lectura Comprensiva de Inglés para Médicos, en una plataforma montada sobre Lotus Notes. El curso tuvo poca acogida y una duración de 2 meses. En 2002 la misma UTPL ofertó un total de 30 cursos y un programa universitario a través de campus virtual (UNESCO, 2004).

La cultura de uso de Internet y su tasa de penetración han incidido en el lento desarrollo de la educación a distancia mediada por tecnologías en Ecuador; sin embargo, la oferta de programas de prestigiosas universidades internacionales obliga a la universidad ecuatoriana a repensar su accionar y a establecer alianzas académicas que le permitan aprovechar su experiencias y su infraestructura, con instituciones como la UNAD.

En conclusión, en Ecuador existen programas acreditados, con los cuales cuenta la UNAD, y, además, puede llegar ofertar, como Psicología, Comunicación Social, Licenciaturas de Inglés como Lengua Extranjera, Etnoeducación, Agronomía, Ingeniera Industrial e Ingeniería Electrónica, todos los cuales gozan de reconocimiento en muchos países miembros y asociados a estos tratados, como Colombia y Ecuador.

\section{Conclusiones}

El $76,6 \%$ de la población educativa del Ecuador prefiere las entidades públicas, y el $23,4 \%$, las privadas.

La totalidad de los estudiantes de institutos superiores técnicos y tecnológicos en Ecuador optan por programas análogos a los ofertados por la UNAD, como Administración y Comercio, en un $40,72 \%$, y por las tecnologías, en un $30,25 \%$.

En Ecuador los sectores productivos, de industria manufacturera, agricultura, comercio, construcción, transporte y enseñanza se pueden considerar nichos de mercados potenciales para los programas académicos de la UNAD.

Se pueden considerar como otros nichos de mercados potenciales los sectores productivos de restaurantes y hotelería; también, los de agricultura y ganadería, electricidad y agua potable, por ser todos ellos importantes generadores de empleo en el Ecuador.

En la investigación se destacan tres tratados o acuerdos comerciales internacionales para la oferta y la movilidad de programas académicos entre Ecuador y Colombia; estos son: el Grupo de Río, el ALADI y el Mercosur.

La infraestructura de acceso a Internet de las universidades es de un $68 \%$, y cuenta con enlaces que van desde los $64 \mathrm{Kbps}$ hasta 1,5 Mbps. Además, las universidades con acceso a Internet cuentan con servidores y correo electrónico, lo que garantiza su presencia en la red con sus respectivas páginas web. 
En Ecuador hay experiencia en la oferta de programas semipresenciales de diplomado con apoyo de la tecnología, con un componente a distancia y con un componente de videoconferencias, por medio de la red de videoconferencia satelital.

En Ecuador existen 19 universidades online, que equivalen al $28 \%$ de las universidades, y el $72 \%$ son presenciales, si bien en algunas se puede mezclar la modalidad semipresencial, para ofertar sus programas.

\section{Referencias bibliográficas}

Álvarez, A. I. (2005). La creación del texto escrito: Composición y uso de modelos de Apuestas Productivas Ecuador. Recuperado de http://www.mcpec.gob. ec/images/stories/noticias/DocumentosSectoriales/subsectoreseconomicos_apuestasproductivas.pdf

Arias, F. G. (2006). Proyecto de Investigación: Introducción a la metodología científica. (5ae ed.) Caracas: Episteme.

Asociación Nacional de Universidades e Instituciones de Educación Superior (ANUIES) - U N E S C O . (2004). La Educación Superior Virtual en América Latina y el Caribe. Recuperado de http://books.google. com.co/books? id=Zh09X8OodyoC\&lpg=PP1\&dq=inaut hor\%3 A\%22Anuies-unesco\%22\&hl=es\&pg=PA6\#v=0 nepage $\& q \&=$ false

Didou, S. (2002). Transnacionalización de la educación superior, aseguramiento de la calidad y acreditación en México. Revista de la Educación Superior en Línea 124.

Didou, S. C., García C. \& Muñoz, L. (2004). Assurance qualité, accréditation de l'enseignement supérieur et certification des compétences en Amérique Latine. París: UNESCO, Second Global Forum on quality assurance, accreditation and capacity building in higher education. Recuperado de http://www.unesco. org. económicamente activa (PEA) del Ecuador. Ecuador: Instituto Nacional de Estadísticas y Censos -INEC-. Recuperado de http://www.inec.gov.ec/web/ guest/publicaciones/estudios/soc/dem_prof.

Estudios Demográficos en Profundidad. (s. f.). Análisis y proyección de la población

Estudios, Títulos y Diplomas de Educación Superior en América Latina y El Caribe. 1974 y Borrador de propuestas y recomendaciones formuladas al tenor de la Reunión Convenio Regional de Convalidación de Estudios, Títulos y Diplomas de Educación Superior en América Latina y El Caribe, realizada en Bogotá.

Recuperado de http://iesalc.unesco.org.ve/pruebaobservatorio/documentos pdf/.
Galarza L, G. (2004). La educación superior no universitaria en Ecuador: observatorio virtual para la educación superior en América Latina y el Caribe (OEVALC). Recuperado de http://unesdoc.unesco.org/ images/0014/001404/140490s.pdf

Gallardo, Y. \& Moreno, A. (1999). Serie Aprender a Investigar: Módulo 3 Recolección de la Información. Colombia: ICFES.

García, C. (2004). Comercialización de la educación superior. Algunas reflexiones para el caso latinoamericano. Revista de la Educación Superior XXXIII (2). México: Asociación Nacional de Universidades e Instituciones de Educación Superior (ANUIES).

Grajales, T. G. (2000). Tipos de Investigación. Recuperado de http://tgrajales.net/investipos.pdf

IESALC. (2003). Internacionalización y nuevos proveedores de educación superior en Ecuador. Recuperado de http://unesdoc.unesco.org/ images/0014/001404/140479s.pdf.

IESALC. (2005). Propuesta de modificación del Convenio Regional de Convalidación de

Instituto Nacional de Estadísticas y Censos -INEC-. (2009). Estudios Demográficos en Profundidad. Análisis y Proyección de la Población Económicamente Activa (PEA) del Ecuador. Recuperado de http:// www.inec.gov.ec/web/guest/publicaciones/estudios/ soc/dem_prof.

Jaramillo, I. C. \& Aponte, C. (2000). Manual para la movilidad académica internacional.

Kotler, P. (1996). Dirección de mercadotecnia, 8va ed. México: Pearson, Prentice Hall.

Kotler, P. (2001). Dirección de Marketing, La Edición del Milenio. México: Pearson, Prentice Hall.

Malo, S. (2006). Créditos académicos (SICA) y Complemento al título (CAT). Recuperado de http:// www.iesalc.unesco.org.ve/pruebaobservatorio/documentos\%20pdf/12\%20reunión\%20convalidación\%20 de\%20diplomas\%20\%20el\%20salvador/creditos_ acade micos\%20sma\%201.pdf

Mercosur Educativo. (2009). Portal del Sector Educativo. Recuperado de http://www.sic.inep.gov.br/

Ministerio de Fomento, Industria y Comercio 2010. Recuperado de http://www.mific.gob.ni/LinkClick.aspx ?fileticket=rYBuyLoLHCM\%3D\&tabid=34 O\&language=en-US

Oportunidad para docentes, investigadores y administrativos de instituciones de educación superior. Bogotá: Red Colombiana de Cooperación Internacional para la Educación Superior-RCI-ASCUN. 
Rama, C. (2009). La tendencia a la masificación de la cobertura de la educación superior en América Latina. Revista Iberoamericana de Educación 50, 173-195.

Sistema de Educación Superior del Ecuador (2006). Consejo Nacional de Educación Superior (CONESUP). Recuperado de http://tuning.unideusto.org/ tuningal/images/stories/presentaciones/ecuador.pdf Texto. España: Ediciones Nobel.
Universidades a Distancia en Ecuador. Recuperado de http://www.altillo.com/universidades/universidades_ ecuol.asp

Weise, C. \& Laguna, J. L. (2009). La educación superior en la Región Andina, Bolivia, Perú y Ecuador. Recuperado de http://iteso.mx/ carlosc/pagina/cursoUAHI/ Conferencias/lectura_en_papel2b.htm 